archives

of thermodynamics

Vol. 38(2017), No. 4, 209-227

DOI: $10.1515 /$ aoter-2017-0032

\title{
Monte Carlo analysis of the battery-type high temperature gas cooled reactor
}

\author{
MARCIN GRODZKI \\ PIOTR DARNOWSKI* \\ GRZEGORZ NIEWIŃSKI
}

Warsaw University of Technology, Institute of Heat Engineering, Nowowiejska 21/25, 00-665 Warsaw, Poland

\begin{abstract}
The paper presents a neutronic analysis of the battery-type 20 MWth high-temperature gas cooled reactor. The developed reactor model is based on the publicly available data being an 'early design' variant of the U-battery. The investigated core is a battery type small modular reactor, graphite moderated, uranium fueled, prismatic, helium cooled high-temperature gas cooled reactor with graphite reflector. The two core alternative designs were investigated. The first has a central reflector and $30 \times 4$ prismatic fuel blocks and the second has no central reflector and $37 \times 4$ blocks. The SERPENT Monte Carlo reactor physics computer code, with ENDF and JEFF nuclear data libraries, was applied. Several nuclear design static criticality calculations were performed and compared with available reference results. The analysis covered the single assembly models and full core simulations for two geometry models: homogenous and heterogenous (explicit). A sensitivity analysis of the reflector graphite density was performed. An acceptable agreement between calculations and reference design was obtained. All calculations were performed for the fresh core state.
\end{abstract}

Keywords: HTGR; HTR; Monte Carlo; Serpent; U-battery; SMR; Battery type

\section{Introduction}

The nuclear power provides over $11 \%$ of the electricity consumed worldwide. There are about 450 operating power reactors and about 60 are

${ }^{*}$ Corresponding Author. Email piotr.darnowski@itc.pw.edu.pl 
under construction [1,2]. Most of currently operating and constructed nuclear power plants (NPPs) are using high power ( 1 GWe) light-water reactors (LWRs). The absence of carbon dioxide emissions, low-cost fuel, high capacity-factor and stable baseload power production are considered as the most important advantages of the nuclear power. In the era of a significant development of the renewable energy sources and gas sources, nuclear power loses its relative importance, especially in the European Union and the United States. However, renewables have various disadvantages, such as the unpredictability of energy production, since gas sources are characterized by problems like large fuel prices and their variations. In some cases, a stable and highly reliable source of nuclear power may be needed. Small modular reactors (SMRs) have a potential to be interesting complementation to the future power grid and those may be even alternative to today's nuclear power. Unfortunately, large-sized reactors have some disadvantages. For instance, a large unit can be built only when high power demand is present and only in proper locations, characterized by sufficient cooling and properly remote. Construction and operation of a large NPP are not always possible since it is a very capital-intensive investment and very complex engineering project. Plenty of those problems do not concern SMR designs. This type of reactor requires a much lower capital investment, potentially simpler to build, the flexible option being less capital-intensive with project management being less demanding and less problematic project size and timespan. Moreover, most of the plant and reactor assembly process can be performed in the factory conditions with simpler assurance of the high manufacturing and safety standards. In fact, the unit costs are expected to be higher but it is mainly because the economy of scale is less important. Nevertheless, it is expected that this drawback will be overwhelmed by other positive effects. The detailed discussions about different aspects of the small modular reactors technology are presented in several reports and papers $[2-6,8,20]$.

The special type of SMRs are battery type reactors, which are characterized by very long fuel cycle length ( $>5$ years) which can be even equal to the core lifetime. Those are usually very small units with the capability of automatic operation. Their size allows construction in places where there is no access to the national power grid, like remote locations or where the power demand is much lower. Battery type SMRs are designed in such a way that each element of the reactor can be delivered by railway, by roadway or by the sea. All those factors significantly increase possible locations 
for utilization of this type of reactor [7]. Some modern SMR designs may provide not only the electricity but the process heat which may be a very interesting option for the industry.

There are many types and designs of SMRs or battery type reactors [5] and one of them are high-temperature gas-cooled reactors (HTGR) $[11,14,15]$. The technology is significantly different from commonly used light water reactors and HTGRs are not commonly used. However, interest in this technology has increased significantly in recent years. One of the most notable advantages of this type of reactor is high core outlet coolant temperature (above $700{ }^{\circ} \mathrm{C}$ ) and it allows to reach higher thermal efficiency than in any traditional reactor. Moreover, high-temperature heat can be used in many industrial processes, e.g., hydrogen production. So far, 7 reactors of this type have been built and 2 of them are still operational today HTR-10 (China) and HTTR (Japan) [8]. At present, one HTR-PM reactor is under construction in China [8]. There are many new designs and projects being developed around the World [2].

In the open literature, there is a description of a very small prismatic battery-type high-temperature helium gas cooled reactor, which can operate up to ten years without refueling. The reactor design is called U-battery and detailed data for several alternative designs are described in the publicly available reports $[7,9]$. The concept has been under development since 2007 by Delft University of Technology and Manchester University and financed by URENCO, Koopman and Witteveen. Currently it is developed by the U-battery consortium in partnership with several companies. It is worth to mention that, the alternative 10 MWth core design is being considered to be built in Poland [21]. The basic aim of this study was to build a numerical model and perform neutronic calculations, data analysis and comparison with literature for the selected U-battery design variant.

\section{Materials and methods}

\subsection{SERPENT computer code}

The SERPENT is a continuous energy neutron transport Monte Carlo computer code which has been developed by Finnish VTT research center since $2004[18,22,23]$. The code was used as a state-of-the-art Monte Carlo tool for reactor physics application with fuel burnup capability. The code is a popular research tool in design and analyses of the novel reactor systems. The code and Monte Carlo method were applied as an excellent tool 
to obtain the effective multiplication factor in complex geometries and its abilities to perform this task may be better than in the case of deterministic tools. All calculations were performed with recent SERPENT version 2.1.28 and two different nuclear data libraries ENDF/B-VII and JEFF-3.1.1 $[18,22]$.

\subsection{The core design and models}

\subsubsection{The basic design}

The considered core is a prismatic-type 20 MWth HTR with TRISO type fuel. The reactor core is composed of several prismatic fuel elements (37 or 30) which are made of identical fuel blocks stacked into four axial levels $(37 \times 4$ or $30 \times 4)$. The reactor uses inert helium as a coolant which has a negligible impact on the neutron economy and with core inlet temperature $250{ }^{\circ} \mathrm{C}$ and outlet temperature $750{ }^{\circ} \mathrm{C}$. The fuel blocks are made of graphite and ceramic materials with high thermal capacity and heat resistance. Thanks to that the core is accident resistant - it has a large time scale of transients and accidents. Moreover, it has low power density which allows in most cases to use only passive coolant systems to remove decay heat. The core has a negative reactivity temperature coefficient - reactor power is decreasing when the temperature in the core is increasing. Electricity generation can be produced in a direct or indirect cycle. It's working as a 'nuclear battery', which means that it can work from 5 to 10 ten years without refueling with high burnup capability ( $\sim 70 \mathrm{GWd} / \mathrm{tHM})$. All core design data used in this paper are based on the Design of U-Battery [7] report and documents related to it $[9,10]$. Various design alternatives of this reactor were considered in those reports.

\subsubsection{TRISO particles and fuel compacts}

The reactor uses TRISO-coated type fuel particles embedded in fuel compacts. Those are concentric spherical particles with the kernel made of uranium dioxide with enrichment equal to $20 \mathrm{wt} \% \mathrm{U}-235$. The kernel is coated by several protective layers. The first layer, buffer, directly covers fuel kernel and is made of low-density pyrocarbon. It is designed to accommodate gaseous fission products and to attenuate fission product recoil from a kernel with fuel. The next layer, inner pyrolytic carbon (IPyC), is made of high-density pyrocarbon and is also designed to hold fission products. The third layer is made of silicon carbide ( $\mathrm{SiC})$ and is the primary 
containment. The last layer is made of pyrocarbon and is called outer pyrolytic carbon (OPyC) layer [11]. Table 1 shows the density of materials and thickness of each layer and fuel compact parameters.

Table 1: TRISO and fuel compact parameters [7].

\begin{tabular}{|l|c|c|}
\hline \multicolumn{1}{|c|}{ TRISO Parameter } & Value & Unit \\
\hline \hline Radius of fuel kernel & 250 & $\mu \mathrm{m}$ \\
\hline Density of fuel kernel & 10.5 & $\mathrm{~g} / \mathrm{cm}^{3}$ \\
\hline Thickness of buffer layer & 100 & $\mu \mathrm{m}$ \\
\hline Density of buffer layer & 1 & $\mathrm{~g} / \mathrm{cm}^{3}$ \\
\hline Thickness of IPyC layer & 35 & $\mu \mathrm{m}$ \\
\hline Density of IPyC layer & 1.9 & $\mathrm{~g} / \mathrm{cm}^{3}$ \\
\hline Thickness of SiC layer & 35 & $\mu \mathrm{m}$ \\
\hline Density of SiC layer & 3.2 & $\mathrm{~g} / \mathrm{cm}^{3}$ \\
\hline Thickness of OPyC layer & 40 & $\mu \mathrm{m}$ \\
\hline Density of OPyC layer & 1.97 & $\mathrm{~g} / \mathrm{cm}^{3}$ \\
\hline \hline Compact Parameter & Value & $\mathrm{Unit}$ \\
\hline \hline Radius of fuel compact & 0.6225 & $\mathrm{~cm}$ \\
\hline Height of fuel compact & 4.93 & $\mathrm{~cm}$ \\
\hline Packing Fraction of TRISO & 0.3 & - \\
\hline
\end{tabular}

The TRISO particles are formed in the so-called fuel compacts and those are characteristic for prismatic type cores. They have a cylindrical shape and comprise with TRISO and graphite matrix. The fifteen compacts are stacked inside the fuel block (column). The amount of fuel is corresponding to the packing fraction equal to $30 \%$ and it is defined as a ratio of the total fuel particles volume to the fuel compact volume (Tabs. 1 and 2).

The geometry of the prismatic HTGR reactor is relatively difficult to model. The most concerning problem is the modelling of TRISO particles embedded in fuel compacts. Two types of geometry models were analyzed in this work. The first is simplified and uses homogenization of the compacts. Hence, the fuel compact is filled with the mixture of the TRISO particle materials and matrix materials. The model is created as follows: first, the exact amount of individual material that is present in the fuel compact volume is calculated. Then the density of each material in fuel compact 


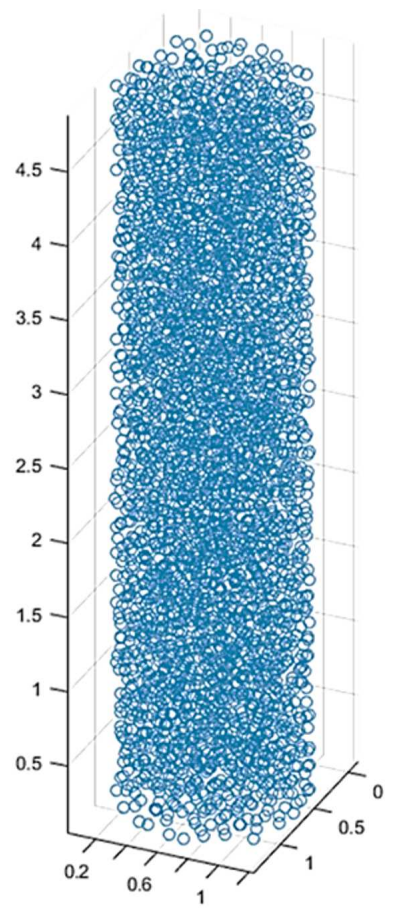

Figure 1: Visualization of the single fuel compact with TRISO particles locations for heterogenous (explicit) geometry model. Units are centimeters.

is calculated: the mass of the material is divided by the volume of fuel compact. Fuel compact is filled by the homogenous material, which is a mixture of all the materials occurring in its volume. Thanks to that, the complicated model with precise positioning of TRISO particles is avoided. As a result, the calculations are made with some degree of simplicity and it causes differences in neutron multiplication properties of the system. Otherwise, this approach was used in the reference report $[7,9]$ and it is popular in some HTGR analyses.

The second approach is the explicit model and it is taking into account random microstructure of the fuel. The Serpent code allows the user to provide particles coordinates and to model precisely the TRISO fuel. In order to create the most accurate model that takes into consideration the positions of TRISO particles, a special dedicated code has been developed that calculates the geometry parameters and generates random particle coordinates. It was evaluated that there should be 4416 TRISO particles 
in each fuel compact (30\% volume fraction) and coordinate points were generated using the code (see Figs. 1 and 2). For simplicity, only one random configuration was used in the SERPENT calculations for all fuel compacts (see Fig. 1). It is the important simplification, the generation of the distinct random patterns for every single fuel compact was considered as complex task beyond the scope of this work. The maximum reactivity uncertainty due to the deviation in the geometry pattern may be estimated to be less than $150 \mathrm{pcm}$ (per cent mile) [25]. This value is the maximum difference between cubic pattern and multiple realizations random pattern for infinite medium described in [25]. What is more, the reference [24] compares cubic pattern with random pattern for a full-core HTR and the deviation is less than $50 \mathrm{pcm}$. However, in this research, the fuel geometry heterogeneity effect [9] onto the neutron multiplication was intended to be shown and estimated quantitively with single random geometry realization.
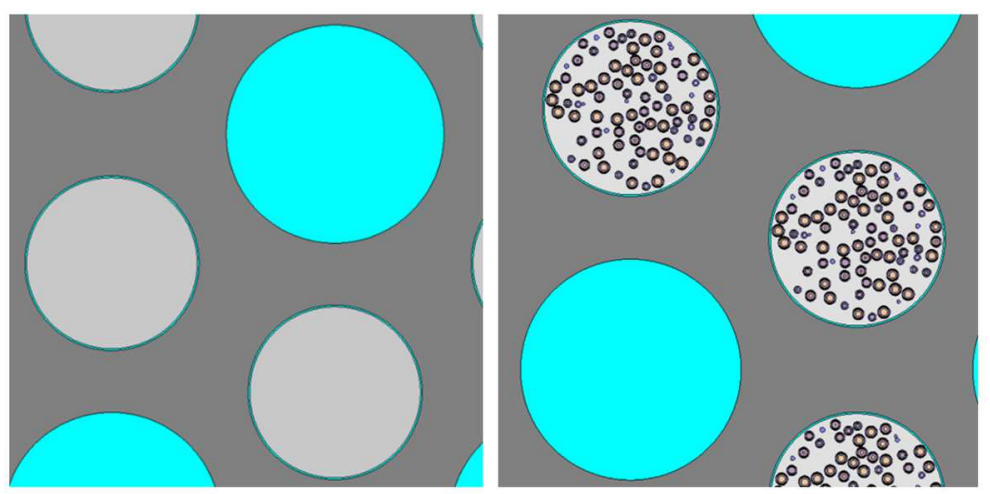

Figure 2: Magnified fuel block cross-section with visible fuel compacts, cooling channels and matrix. Homogenized geometry (left) and heterogenous explicit type geometry with TRISO particles (right).

\subsubsection{Fuel-blocks}

The hexagonal (prismatic) blocks made of graphite are stacked to form a fuel element (Tab. 2). Particularly, four fuel blocks are set tight side by side. Each block contains graphite matrix, fuels channels (rods) filled with fuel compacts and helium coolant channels (holes). Additionally, in the proposed fuel design, there are six fixed burnable poison (FBP) channels [9]. The calculations which are the main part of this report, as well as 
reference results are taken from the literature [9], relating to fuel blocks with FBP replaced by fuel compacts. There is also a special type of block, entirely made of the graphite which works as a reflector. The basic fuel block design is based on GT-MHR fuel block as described in [12]. Figures 3 and 4 presents the vertical and horizontal cross-section for the fuel block SERPENT models.

Table 2: Basic parameters of fuel block [9].

\begin{tabular}{|l|c|c|}
\hline \multicolumn{1}{|c|}{ Parameter } & Value & Unit \\
\hline \hline Width flat-to-flat & 36 & $\mathrm{~cm}$ \\
\hline Height of the block & 80 & $\mathrm{~cm}$ \\
\hline Number of fuel channels & 216 & - \\
\hline Radius of fuel channel & 0.635 & $\mathrm{~cm}$ \\
\hline Number of coolant channels & $102 / 6$ & - \\
\hline Radius of coolant channel & $0.794 / 0.635$ & $\mathrm{~cm}$ \\
\hline Number of fixed burnable (FBP) holes & 6 & - \\
\hline Radius of FBP hole & 0.635 & $\mathrm{~cm}$ \\
\hline Number of fuel compacts per one channel & 15 & - \\
\hline Number of fuel blocks per fuel element & 4 & - \\
\hline
\end{tabular}

\subsubsection{The core design and full core models}

There are two core configurations considered, the first case has $37(37 \times 4)$ blocks per core level and the second $30(37 \times 4)$ blocks. The main difference is due to seven central fuel blocks which are replaced by reflector blocks in the second case. Figures 5 and 6 present SERPENT model's visualizations for both core configurations. Fuel blocks are surrounded by the permanent side reflector and its outer diameter is $3.1 \mathrm{~m}$. The thickness of the side reflector depends on the outer diameter of the reactor vessel. There are other layers between side reflector and RPV as described in design reports but those were not modelled. Figures 5 and 6 (right) show top and bottom reflectors modelled by $0.5 \mathrm{~m}$ high graphite layers. Coolant channels run through reflectors in accordance with blocks channels. It should be noted that the whole geometry is modelled only until the side reflector as in reference calculations [7]. 

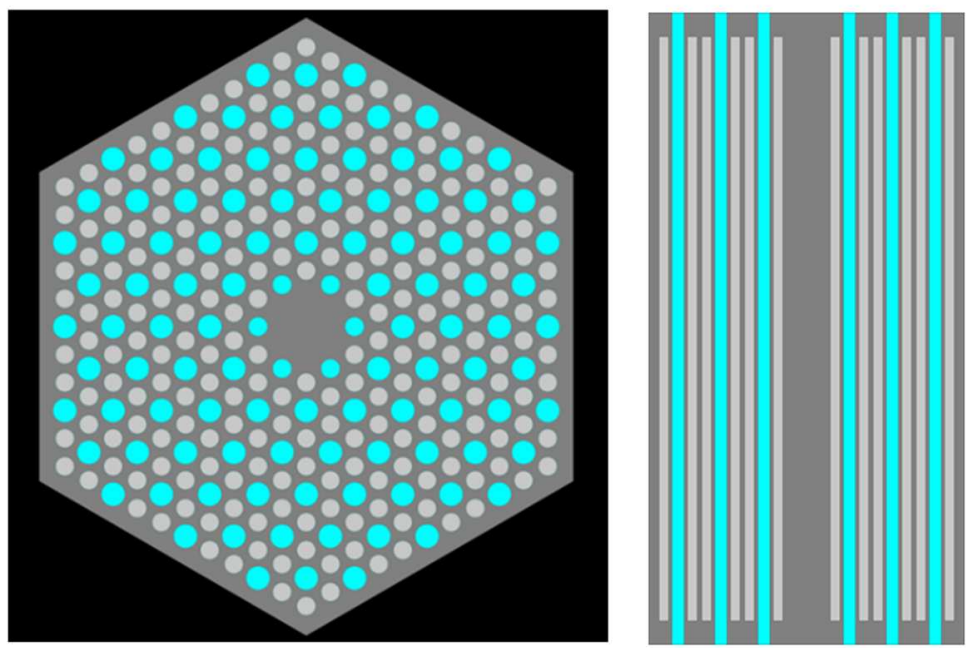

Figure 3: Fuel block homogenous type, horizontal cross-section (left) and vertical crosssection (right).
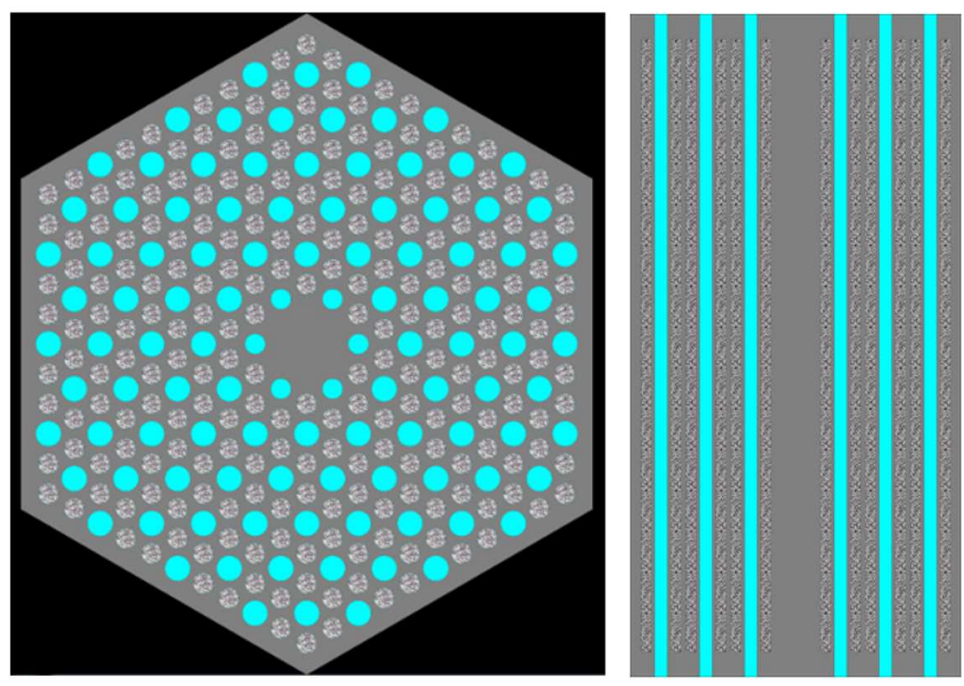

Figure 4: Fuel block horizontal cross-section, explicit type geometry (left) and vertical cross-section (right). 

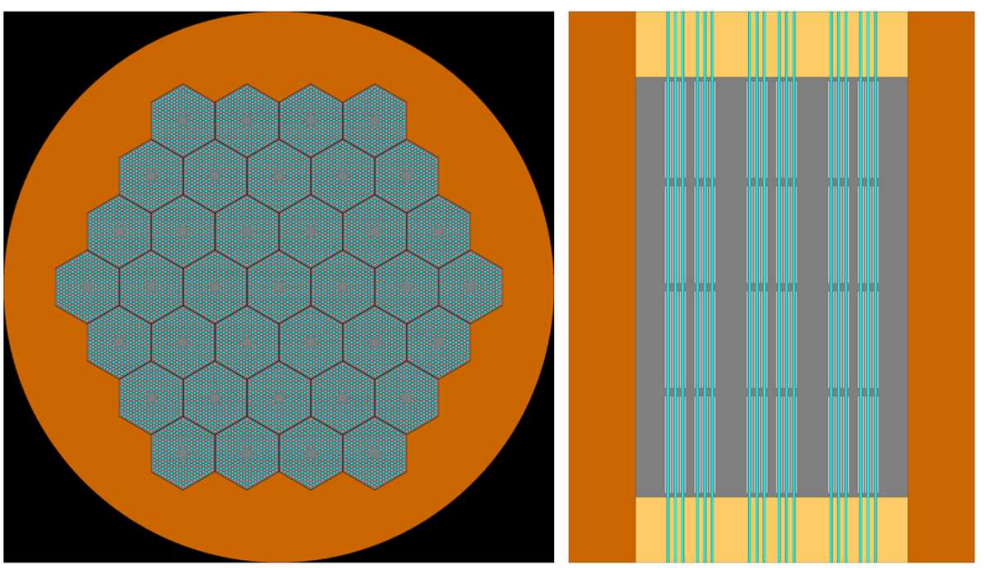

Figure 5: Full-core model horizontal cross-section for $37 \times 4$ reactor, explicit type geometry (left) and vertical cross-section (right).
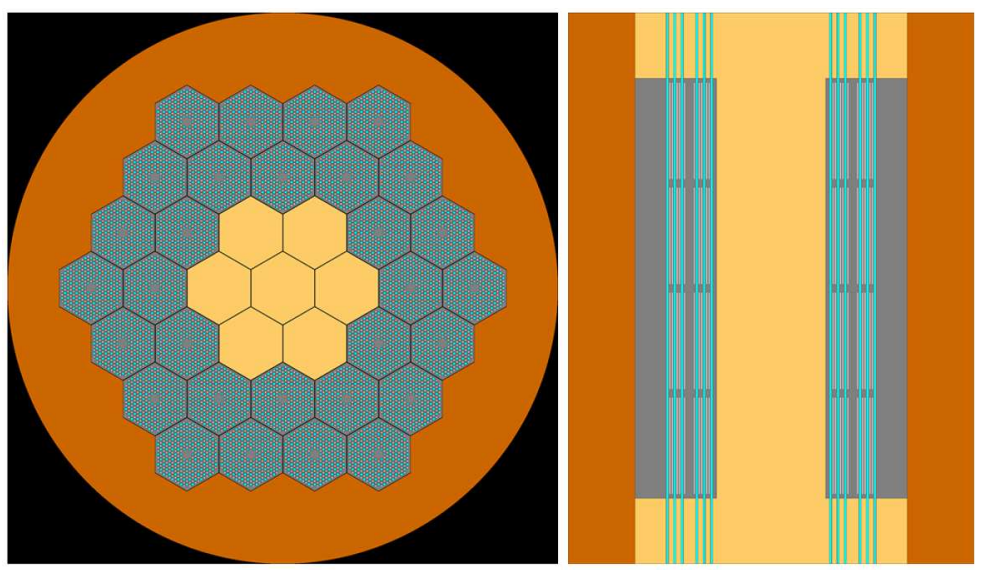

Figure 6: Full-core model horizontal cross-section for $30 \times 4$ reactor, explicit type geometry (left) and vertical cross-section (right).

The graphite densities applied in calculations are presented in Tab. 3. Due to the lack of precise data concerning the graphite densities for core structures, certain values were based on data available in the following references: $[13,12,16,17]$. It was also the motivation to perform, additional sensitivity calculations related to reflector's graphite density. 
Table 3: Graphite densities for different reactor components.

\begin{tabular}{|l|l|l|}
\hline \multicolumn{1}{|c|}{ Name } & Value & Unit \\
\hline \hline Permanent reflector graphite & 1.73 & $\mathrm{~g} / \mathrm{cm}^{3}$ \\
\hline Reflector block graphite & 1.78 & $\mathrm{~g} / \mathrm{cm}^{3}$ \\
\hline Fuel block graphite & 1.85 & $\mathrm{~g} / \mathrm{cm}^{3}$ \\
\hline Top and bottom reflector graphite & 1.78 & $\mathrm{~g} / \mathrm{cm}^{3}$ \\
\hline Fuel compact graphite & 1.2 & $\mathrm{~g} / \mathrm{cm}^{3}$ \\
\hline Reflector's graphite density for additional calculations & 2.3 & $\mathrm{~g} / \mathrm{cm}^{3}$ \\
\hline
\end{tabular}

\subsection{Investigated cases}

The first group of analyzed cases: $0 \mathrm{~A}$ and $0 \mathrm{~B}$ (Tab. 4), covers single fuel block calculations with the estimation of the infinite multiplication factor (see Figs. 3 and 4). Reflective boundary conditions were applied on all outer boundary surfaces. Simulations were performed with 20000 neutrons per 500 active cycles and 100 inactive cycles. The second group, (Tab. 3 ) covers full core cases (see Figs. 5 and 6), and for all of them black boundary conditions were applied, and simulations were performed with 200000 neutrons per cycle, 500 active and 100 inactive cycles. The selected neutron populations and cycles were based on the recommendations and typical approach in Monte Carlo criticality analysis [26].

Table 4: List of the basic cases analyzed with SERPENT.

\begin{tabular}{|l|l|}
\hline Case & \multicolumn{1}{|c|}{ Description } \\
\hline \hline 0A & single fuel block, homogenous type \\
\hline OB & single fuel block, explicit type \\
\hline IA & $37 \times 4$ full core, homogenous type \\
\hline IB & $37 \times 4$ full core, explicit type \\
\hline IIA & $30 \times 4$ full core, homogenous type \\
\hline IIB & $30 \times 4$ full core, explicit type \\
\hline
\end{tabular}

The current state-of-the-art Monte Carlo criticality simulations involve studies of the fission source convergence and it covers the multiplication 
factor (eigenvalue) and Shannon Entropy investigation [19]. Those parameters were examined for all models and satisfactory convergence was achieved with 100 inactive cycles.

The single fuel block models, both homogenized and explicit cases and full core homogenized cases were compared with reference results. Those were obtained with SCALE5.1 or SCALE6 package with KENO Monte Carlo solver and were taken from reference [9] and are reproduced in Tab. 5. Worth to highlight is the fact that the explicit model reference results cover only the single fuel block case. Monte Carlo standard deviations were reported only for the full-core calculations $(\sim 130 \mathrm{pcm})$.

Table 5: Reference values obtained with SCALE/KENO code. Taken from [9].

\begin{tabular}{|c|c|c|c|c|}
\hline $\begin{array}{l}\text { Ref. } \\
\text { case }\end{array}$ & Type & Code & $k_{e f f}{ }^{*}$ & $\sigma^{*}$ \\
\hline $0 \mathrm{~A}$ & $\begin{array}{l}\text { fuel block, homoge- } \\
\text { nous model }\end{array}$ & Scale 6 & 1.3945 & $\mathrm{~N} / \mathrm{A}$ \\
\hline OB & $\begin{array}{l}\text { fuel block, explicit } \\
\text { model }\end{array}$ & Scale 6 & 1.4456 & $\mathrm{~N} / \mathrm{A}$ \\
\hline IA & $\begin{array}{l}\text { reactor } 37 \times 4 \text { type, } \\
\text { homogenous model }\end{array}$ & Scale 5.1 & 1.2980 & 0.0017 \\
\hline IIA & $\begin{array}{l}\text { reactor } 30 \times 4 \text { type, } \\
\text { homogenous model }\end{array}$ & Scale 5.1 & 1.3397 & 0.0017 \\
\hline
\end{tabular}

Information about reflector's graphite density was missing in the reference documentation $[7,9]$. Additional sensitivity calculations were performed for the full core cases IA, IB, IIA and IIB. Hence, two variants of reflector density were calculated: the first variant assumed values consistent with the industrial grade graphite. In the second variant reflector's graphite density was assumed to be $2.3 \mathrm{~g} / \mathrm{cm}^{3}$, which is equal to anisotropic graphite's density. The new reflector density is the only change in comparison to other cases' parameters. The idea of performing a calculation for alternative reflector's density comes from the reference [13], the oldest document containing information about the design.

In general, the reactivity differences were calculated according to the formula (in units of pcm)

$$
\Delta \rho=\frac{k_{1}-k_{0}}{k_{1} k_{0}} \times 10^{5}
$$


and the relative (percent) difference between the results by the following formula:

$$
\mathrm{RD}=\frac{k_{1}-k_{0}}{k_{1}} \times 100,
$$

where $k_{1}$ and $k_{0}$ are neutron multiplication factors (eigenvalues) for compared cases.

\section{$3 \quad$ Results and discussion}

\subsection{Single block results}

Table 6 shows the results of the infinite multiplication factor, $k_{i n f}$, calculations for the 0A (fuel block, homogenous type) and 0B (fuel block, explicit type) cases compared with reference values. This is the only chance where comparison of the reference values for the explicit and homogenous model for the same cases was possible.

Table 6: Comparison of the SERPENT and reference results [9] for fuel single blocks calculations.

\begin{tabular}{|c|c|c|c|c|c|c|c|}
\hline Case & Type & Lib. & $\begin{array}{c}k_{i n f} \\
\text { SERPENT }\end{array}$ & $\begin{array}{c}\sigma \text { SERPENT } \\
(\mathrm{pcm})\end{array}$ & $\begin{array}{c}k_{\text {inf }} \text { ref. } \\
\text { value }\end{array}$ & $\begin{array}{c}\Delta \rho \\
(\mathrm{pcm})\end{array}$ & $\mathrm{RD}, \%$ \\
\hline \hline 0A & Homogenous & ENDF & 1.39323 & $0.00017(12)$ & 1.3945 & 65 & 0.09 \\
\hline 0A & Homogenous & JEFF & 1.39288 & $0.00018(13)$ & 1.3945 & 83 & 0.12 \\
\hline 0B & Explicit & ENDF & 1.44871 & $0.00028(19)$ & 1.4456 & -149 & -0.22 \\
\hline 0B & Explicit & JEFF & 1.44847 & $0.00016(11)$ & 1.4456 & -137 & -0.20 \\
\hline
\end{tabular}

The results obtained using the JEFF library are slightly lower than those calculated with the ENDF library with the difference less than $<20 \mathrm{pcm}$ for both explicit and homogenous geometry models. SERPENT calculations are close to the reference results for both libraries with reactivity differences being less than $150 \mathrm{pcm}$ (see Tab. 6). The minor difference in $k_{\text {inf }}$ are found for the homogenous model (Case 0A) and the slightly larger difference was found for the explicit model (Case 0B). What is more, it was observed that the infinite multiplication factors calculated for the explicit models are higher than for the homogenous models by about $\sim 2800 \mathrm{pcm}$ and similar differences are present for reference calculations. In consequence, it can be observed that the heterogeneity effect is strong in terms 
of reactivity and the SERPENT code is able to reproduce it.

The SERPENT calculated Monte Carlo relative statistical errors (Tab. 6) are characterized by standard deviations smaller than $20 \mathrm{pcm}$. On the contrary the KENO statistical errors were not reported [9] and it is not possible to assess the effects of the Monte Carlo uncertainty. Otherwise, taking into account the nature and practice of neutronic simulations with different codes for such complex systems the results with about $\sim 150 \mathrm{pcm}$ deviation are satisfactory and with $\sim 50 \mathrm{pcm}$ are considered as very good. Larger differences for the explicit model are probably caused by the application of the different random geometry pattern.

\section{$3.2 \quad$ Full-core results}

Table 7 shows the results of the effective multiplication factor calculations for homogenous full-core models: IA $(37 \times 4)$ and IIA $(30 \times 4)$ cases compared to the homogenous full-core reference values (see Tab. 5). What is more, Tab. 7 shows a comparison of the basic cases with low (standard) reflector density and sensitivity cases (with asterisk*) with large reflector density.

Table 7: Comparison of the full-core SERPENT results with reference results for homogenous models.

\begin{tabular}{|l|l|l|l|l|l|l|l|}
\hline Case & Lib. & $\begin{array}{l}k_{\text {eff }} \\
\text { SERPENT }\end{array}$ & $\begin{array}{l}\sigma \text { SERPENT } \\
(\mathrm{pcm})\end{array}$ & $\begin{array}{l}\mathrm{k}_{\text {eff }}, \text { ref. } \\
\text { value }\end{array}$ & $\begin{array}{l}\sigma \quad \text { ref. } \\
(\mathrm{pcm})\end{array}$ & $\begin{array}{l}\Delta \rho \\
(\mathrm{pcm})\end{array}$ & $\mathrm{RD}, \%$ \\
\hline \hline \multicolumn{7}{|c|}{ Base case: standard reflector graphite density } \\
\hline IA & ENDF & 1.26502 & $0.00010(8)$ & 1.2980 & 131 & 2009 & 2.54 \\
\hline IA & JEFF & 1.26476 & $0.00010(8)$ & 1.2980 & 131 & 2025 & 2.56 \\
\hline IIA & ENDF & 1.30756 & $0.00009(7)$ & 1.3397 & 127 & 1835 & 2.40 \\
\hline IIA & JEFF & 1.30729 & $0.00010(8)$ & 1.3397 & 127 & 1851 & 2.42 \\
\hline \multicolumn{7}{|c|}{ Sensitivity case: modified reflector graphite density $2.3 \mathrm{~g} / \mathrm{cc}$} \\
\hline IA* & ENDF & 1.29725 & $0.00010(8)$ & 1.2980 & 131 & 45 & 0.06 \\
\hline IA* & JEFF & 1.29691 & $0.00010(8)$ & 1.2980 & 131 & 65 & 0.08 \\
\hline IIA $^{*}$ & ENDF & 1.33924 & $0.00013(10)$ & 1.3397 & 127 & 26 & 0.03 \\
\hline IIA* & JEFF & 1.33898 & $0.00010(8)$ & 1.3397 & 127 & 40 & 0.05 \\
\hline
\end{tabular}


The base case SERPENT results with standard reflector density are up to $2000 \mathrm{pcm}$ lower than reference results obtained with SCALE/KENO. In consequence, it led us to the conclusion that there are very significant differences in modelling. Several potential sources of differences were studied but the reflector density was identified as the most probable.

Table 6 shows that sensitivity cases results are very close to the reference homogenous results. Differences in reactivity are $26-65 \mathrm{pcm}$ and relative difference is less than $0.1 \%$. This allows us to conclude that there is a high probability that the reflector's graphite density was equal to (or close) $2.3 \mathrm{~g} / \mathrm{cc}$ and this value has been used in reference calculations. However available data and performed calculations cannot prove with certainty such a hypothesis over reasonable doubt. Otherwise, statistical errors of the IA* and IIA* cases (Tab. 6) are 2 to 5 times larger than differences expressed in terms of reactivity. Hence, from the Monte Carlo uncertainty point of view, those results are convergent. Nevertheless, SERPENT calculations were performed with substantially larger neutron population and the statistical errors are an order of magnitude lower than reference results.

Table 8 shows the differences between the explicit (B) and homogenous (A) geometry models for base cases and sensitivity cases $\left(^{*}\right)$. In general, the effective multiplication factors calculated with the explicit model are higher by about $\sim 3000 \mathrm{pcm}$ and $\sim 1800 \mathrm{pcm}$ in the base and sensitivity cases respectively. Hence, as expected, similarly to the single assembly calculations heterogeneity effects are strong. The differences for sensitivity cases are in general 100-200 pcm lower than base cases. Worth to mention that the differences between calculations of the same case and geometry model but using different nuclear libraries are small $\leq 20$ pcm for all cases (Tabs. 7 and 8). Example fission power and thermal flux intensities distribution for homogenous cases are presented in Fig. $7(37 \times 4)$ and $8(30 \times 4)$ [18].

\section{Conclusions and summary}

The obtained multiplication factors for the single prismatic block and the full-core sensitivity cases are considered as being in very good agreement (Tabs. 6 and 7) with the reference data [9]. On the contrary, the full-core base cases with lower reflector density diverge significantly $(\sim 2000 \mathrm{pcm})$. The difference of $<200 \mathrm{pcm}$ may be considered as a satisfactory result. It is especially due to the fact that the models were prepared using the limited design data available in the public reports, the nature of this type 
Table 8: Comparison of the full-core SERPENT results for homogenous and explicite models.

\begin{tabular}{|c|c|c|c|c|c|c|c|}
\hline & \multicolumn{2}{|c|}{ Case $\mathrm{A}$ - homogenous } & \multicolumn{2}{|c|}{ Case B - explicit } & \multirow[b]{2}{*}{$\begin{array}{l}\Delta \rho \\
(\mathrm{pcm})\end{array}$} & \multirow[b]{2}{*}{$\mathrm{RD}, \%$} \\
\hline Case & Lib. & $k_{e f f, h o m}$ & $\sigma$ & $k_{e f f, \exp }$ & $\sigma$ & & \\
\hline \multicolumn{8}{|c|}{ Base case: standard reflector density } \\
\hline I & ENDF & 1.26502 & 0.00010 & 1.31654 & 0.00010 & 3093 & 3.91 \\
\hline $\mathrm{I}$ & JEFF & 1.26476 & 0.00010 & 1.31617 & 0.00010 & 3088 & 3.91 \\
\hline II & ENDF & 1.30756 & 0.00010 & 1.34052 & 0.00010 & 1880 & 2.46 \\
\hline II & JEFF & 1.30729 & 0.00010 & 1.34037 & 0.00010 & 1887 & 2.47 \\
\hline \multicolumn{8}{|c|}{ Sensitivity case: modified reflector density $2.3 \mathrm{~g} / \mathrm{cc}$} \\
\hline $\mathrm{I}^{*}$ & ENDF & 1.29725 & 0.00010 & 1.34730 & 0.00010 & 2864 & 3.71 \\
\hline $\mathrm{I}^{*}$ & JEFF & 1.29691 & 0.00010 & 1.34716 & 0.00010 & 2876 & 3.73 \\
\hline $\mathrm{II}^{*}$ & ENDF & 1.33924 & 0.00013 & 1.37207 & 0.00010 & 1787 & 2.39 \\
\hline $\mathrm{II}^{*}$ & JEFF & 1.33898 & 0.00010 & 1.37163 & 0.00009 & 1778 & 2.38 \\
\hline
\end{tabular}
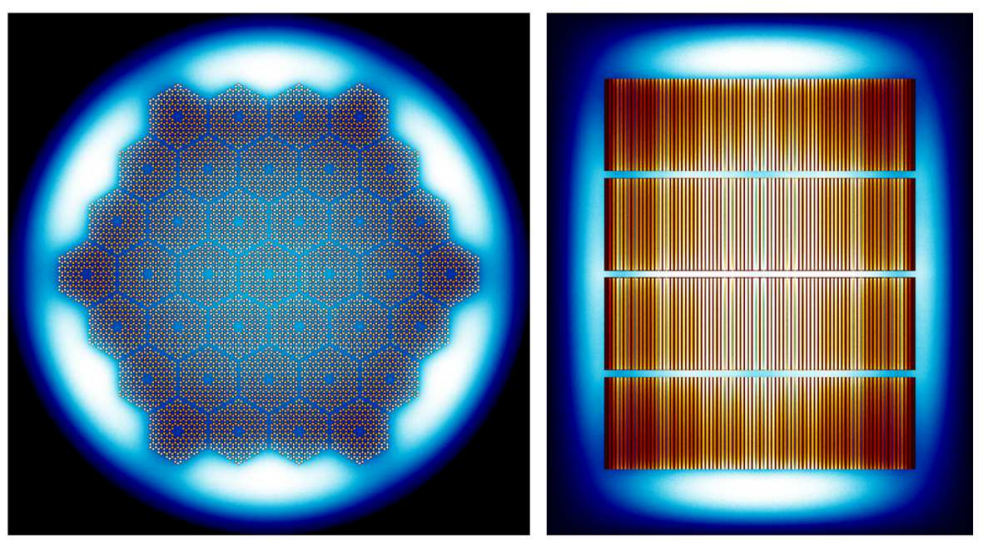

Figure 7: The thermal power and thermal flux distributions in Case IA (homogenous). Horizontal (left) and vertical (right).

of reports and fact that all nuclear number densities were calculated independently. Moreover, the reference results were calculated by different computer code (SCALE/KENO) and those were characterized by a relatively large statistical error. Worth to mention that, a smaller accuracy 

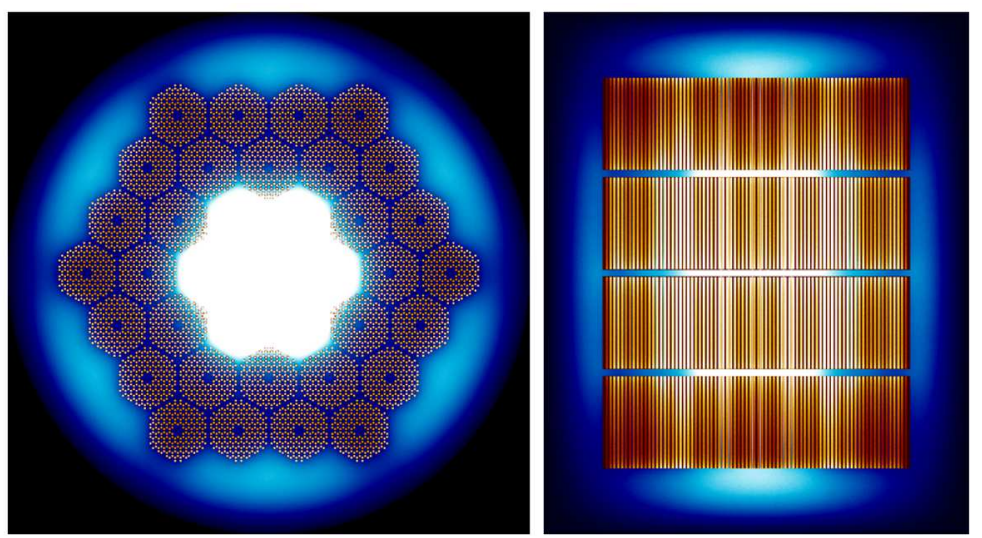

Figure 8: The thermal power and thermal flux distributions in Case IIA (homogenous). Horizontal (left) and vertical (right).

may be found in some reactor physics international benchmark exercises with detailed specifications provided.

Two types of geometrical models were analyzed, homogenous and explicit. In the homogenous model, all materials exist as a mixture in fuel compact volume. In the case of explicit model fissile material is located in clusters consisting of TRISO particles and it is random heterogenous material. Table 6 provides a basis to conclude that the single assembly models both homogenized and explicit were created properly. The larger differences in the explicit case are probably caused by the different random geometry pattern applied in reference calculations. Otherwise, single block models were appropriate to use them to create full-core models. The full-core homogenous model results were consistent with reference results. Unfortunately, explicit full-core reference results were not available.

The fuel geometry heterogeneity effects were observed and compared quantitively. In the case of full-core calculations substantially higher results came from the explicit model $(\sim 1800-3000 \mathrm{pcm})$. Similar effects were observed for a single block $(\sim 2800 \mathrm{pcm})$ and described in the literature [9]. Comparison of reference values resulting from the calculation for both explicit and homogenous models was only possible in one case - in a case of single fuel block calculations. It is because reference reports do not contain full-core explicit calculations for investigated cores. Hence, fullcore explicit results presented in this report are novel for the investigated design. Nevertheless, the further study of the random explicit geometry 
modelling is recommended for the future activities. The application of the single geometry realization may be questionable from the purely theoretical point of view as the selected realization may not be representative. Otherwise, the main purpose of the explicit full-core calculations was achieved, the heterogeneity effect was estimated quantitively and differences are consistent with single block heterogeneity impact.

All investigated cases were calculated using two types of libraries JEFF and ENDF as a standard approach in neutronic calculations. Results from calculations using JEFF library were in every case slightly lower than for ENDF library. The difference is due to the very complex nature of nuclear data and it occurs in any reactor analysis.

Acknowledgments We would like to deeply acknowledge Jakko Leppänen and VTT for the creation of the excellent SERPENT computer code. We would like to state, no conflict of interest with any company, organization and institution. This research was performed as independent university research and was not financed by any organization.

Received 11 October 2017

\section{References}

[1] World Nuclear Association: http://www.woorld-nuclear.org/information-library/ current-and-future-generation/nuclear-power-in-the-world-today.aspx (accessed 5 Oct. 2017).

[2] Nuclear Energy Institute: https://www.nei.org/Knowledge-Center/NuclearStatistics/World-Statistics (accessed 05 Oct. 2017).

[3] International Nuclear Energy Agency: Advances in small modular reactor technology developments. IAEA, Vienna 2014.

[4] International Nuclear Energy Agency: Approaches for Assessing the Economic Competitiveness of Small and Medium Sized Reactors. IAEA Nuclear Energy Ser. NPT-3.7, 2013.

[5] Rowiński M.K., White T.J., Zhao J.: Small and medium sized reactors (SMR): A review of technology. Renewable and Sustainable Energy Rev. 44(2015), 643-656.

[6] NNL: Small Modular Reactors (SMR) Feasibility Study. National Nuclear Laboratory Rep., Dec. 2014.

[7] Ding M., Kloosterman J.L., Kooijman T., Linssen R., Abram T., Marsden B., Wickham T.: Design of U-Battery. Delft, 2011.

[8] World Nuclear Association: http://www.world-nuclear.org/information-library/ nuclear-fuel-cycle/nuclear-power-reactors/small-nuclear-power-reactors.aspx (accessed 05 Oct. 2017). 
[9] Ding M., Kloosterman J.L.: Neutronic feasibility design of a small long-life HTR. Nucl. Eng. Des. 241(2011), 12, 5093-5103.

[10] Ding M., Kloosterman J.L.: Thermal-hydraulic design and transient evaluation of a small long-life HTR. Nucl. Eng. Des. 255(2013), 347-358.

[11] High temperature Gas Cooled Reactor Fuels and Material. IAEA, 2010.

[12] Gas turbine-modular helium reactor (GT-MHR) conceptual design description report. Tech. Rep., General Atomics, 1996.

[13] ZwaAn S.J. DE: Feasibility Study of the U-Battery, PNR-131-2007-004, 2007.

[14] History and Evolution of the HTGR. General Atomic, 2007.

[15] IAEA Current status and future development of modular high temperature gas cooled reactor technology. IAEA, 2001.

[16] Prismatic coupled neutronic/thermal fluids transient benchmark of the MHTGR MW core design, Benchmark definition 2011.

[17] Ortensi J., Cogliati J.J., Pope M.A., Bess J.D., Ferrer R.M., Bingham A.A., Ougouag A.M.: Deterministic Modeling of the High Temperature Test Reactor. INL Rep., 2010.

[18] Leppënen J.: Serpent - A Continuous-Energy Monte Carlo Reactor Physics Burnup Calculation Code, Manual, 2015.

[19] Kaltiaisenaho T.: Statistical Tests and the Underestimation of Variance in Serpent 2, Res. Rep., 2014.

[20] Samul K., Strupczewski A., Wrochna G.: Small Modular Reactors (SMR). Rap. National Centre for Nuclear Research, Świerk 2013.

[21] National Centre for Nuclear Research: https://www.ncbj.gov.pl/pl/aktualnosci/ncbjplanuje-budowe-badawczego-reaktora-nowej-generacji (accessed 05 Oct. 2017).

[22] Leppënen J.: Development of a New Monte Carlo Reactor Physics Code. D.Sc. Thesis, VTT Publication 640, Helsinki University of Technology, 2007.

[23] SERPENT Monte Carlo Code Development Status: http://montecarlo.vtt.fi/development (accessed 30 Aug. 2017).

[24] Stanisz P., Malicki M., Kopeć M.: Validation of VHTRC calculation benchmark of critical experiment using the MCB code. SEED 2016 Conf., E3S Web of Conf. 10, 00123, 2016.

[25] Brown F.B., Matrin W.R., Jei W., Conlin J.L., Lee J.C.: Stochastic Geometry and HTGR Modeling with MCNP5. Los Alamos National Laboratory Rep. LA-UR04-8668, 2005.

[26] Brown F.B.: A Review of Best Practices for Monte Carlo Criticality Calculations. Los Alamos National Laboratory Rep. LA-UR-09-03134, 2009. 